\title{
Solution Estimates for some Weakly Nonlinear ODEs
}

D. BUSKE ${ }^{1}$ Programa de Pós-Graduação em Engenharia Mecânica, UFRGS, Av. Sarmento Leite 425, 90050-170 Porto Alegre, RS, Brasil

J.P. ZINGANO², Programa de Pós-Graduação em Mat. Aplicada, UFRGS, Av. Bento Gonçalves 9500, 91509-900 Porto Alegre, RS, Brasil.

\begin{abstract}
We derive a few fundamental estimates for solutions $\boldsymbol{u}$ of weakly nonlinear ODE systems of the form

$$
\boldsymbol{u}_{t}=\mathrm{A} \boldsymbol{u}+B(t) \boldsymbol{u}+\varepsilon \boldsymbol{f}(t, \boldsymbol{u})+\boldsymbol{g}(t), \quad t>t_{0},
$$

where $\mathrm{A}$ is a constant $n \times n$ matrix all of whose eigenvalues have negative real part and $\varepsilon \boldsymbol{f}$ is suitably small, with $B \in L^{p}\left(t_{0}, \infty\right), \boldsymbol{g} \in L^{q}\left(t_{0}, \infty\right)$ for some $1 \leq p, q \leq \infty$. Our analysis improves and extends some well known results obtained elsewhere for important families of equations within this class.
\end{abstract}

\section{Introduction}

Stability results for weakly nonlinear systems of ODEs can be traced back to late nineteen century, with the fundamental pioneering work of Routh, Poincaré, Lyapunov and others, see e.g. [6], [9], [10], [11]. For example, the Poincaré-Lyapunov theorem assures asymptotic stability for the zero solution of

$$
\boldsymbol{u}_{t}=\mathrm{A} \boldsymbol{u}+B(t) \boldsymbol{u}+\boldsymbol{f}(t, \boldsymbol{u}), \quad t>t_{0}
$$

for sufficiently small initial states $\boldsymbol{u}\left(t_{0}\right)$ when all the eigenvalues of $A$ lie in the left half plane, provided that $\|B(t)\| \rightarrow 0$ as $t \rightarrow \infty$ and $\boldsymbol{f}(t, \boldsymbol{u}) /|\boldsymbol{u}|_{E} \rightarrow \mathbf{0}$ as $|\boldsymbol{u}|_{E} \rightarrow 0$, uniformly in $t$, see [2], [7], [12]. Here, $\|\cdot\|$ denotes some (arbitrary) matrix norm, which for definiteness we will choose hereafter to be the spectral norm,

$$
\|\mathrm{B}\|=\sup _{|\mathbf{v}|_{E}=1}|\mathrm{~B} \mathbf{v}|_{E}, \quad \mathrm{~B} \in \mathbb{C}^{n \times n},
$$

where $|\mathbf{v}|_{E}$ denotes the Euclidean size of $\mathbf{v}=\left(v_{1}, \ldots, v_{n}\right),|\mathbf{v}|_{E}=\sqrt{\left|v_{1}\right|^{2}+\ldots+\left|v_{n}\right|^{2}}$. Such results are now routinely discussed in standard ODE courses making use of a very convenient device, the so-called Gronwall's lemma [4], [5], [7], [12]. We will likewise use this lemma (and some variants thereof) to extend the Poincaré-Lyapunov theorem to more general settings, as described next.

\footnotetext{
${ }^{1}$ Daniela.Buske@bol.com.br, partially supported by CNPq

${ }^{2}$ Jzingano@terra.com.br, partially supported by CNPq \# RD 303563/2003-6
} 


\section{Main Results}

Our goal is to obtain solution bounds for the broader class of nonlinear systems

$$
\boldsymbol{u}_{t}=\mathrm{A} \boldsymbol{u}+B(t) \boldsymbol{u}+\varepsilon \boldsymbol{f}(t, \boldsymbol{u})+\boldsymbol{g}(t), \quad t>t_{0},
$$

with $\boldsymbol{u}\left(t_{0}\right) \in \mathbb{R}^{n}$ given, where A satisfies, as before,

$$
\operatorname{Re} \lambda<0, \quad \forall \lambda \in \operatorname{Spec}(\mathrm{A})
$$

and $B(\cdot)$ is some measurable matrix-valued function such that either $B(\cdot) \in L^{p}\left(t_{0}, \infty\right)$ for some $1 \leq p<\infty$, i.e.,

$$
\int_{t_{0}}^{\infty}\|B(\tau)\|^{p} d \tau<\infty, \quad 1 \leq p<\infty
$$

or else we have $B \in L^{\infty}\left(t_{0}, \infty\right)$ with

$$
\|B(t)\| \rightarrow 0 \quad \text { as } t \rightarrow \infty
$$

In (2.1), we also assume that

$$
\boldsymbol{g} \in L^{q}\left(t_{0}, \infty\right) \quad \text { for some } 1 \leq q \leq \infty
$$

and that $\boldsymbol{f}(\cdot, \cdot)$ is continuous and such that, for each $\gamma>0$, a constant $\Gamma=\Gamma(\gamma)>0$ can be found giving

$$
|\boldsymbol{f}(t, \mathbf{u})|_{E} \leq \Gamma(\gamma)|\mathbf{u}|_{E}, \quad \forall \mathbf{u} \text { with }|\mathbf{u}|_{E} \leq \gamma
$$

Choosing $\sigma>0$ such that

$$
\operatorname{Re} \lambda<-\sigma, \quad \forall \lambda \in \operatorname{Spec}(\mathrm{A}),
$$

we can then state the following result.

Theorem 2.1. Given any $V_{0}, G_{0}>0$, there exist positive constants $K=K\left(V_{0}, G_{0}\right)$, $\varepsilon_{0}=\varepsilon_{0}\left(V_{0}, G_{0}\right)$, depending only on $\mathrm{A}, B, \boldsymbol{f}, \sigma$ and $V_{0}, G_{0}$ above, such that, for each $1 \leq q \leq \infty,\left|\mathbf{u}_{0}\right|_{E} \leq V_{0}$, and $\varepsilon, \boldsymbol{g}$ with

$$
|\varepsilon| \leq \varepsilon_{0}\left(V_{0}, G_{0}\right), \quad \boldsymbol{g} \in \mathcal{G}_{0}(q):=\left\{\mathbf{g} \in L^{q}\left(t_{0}, \infty\right):\|\mathbf{g}\|_{L^{q}\left(t_{0}, \infty\right)}<G_{0}\right\}
$$

the solution $\boldsymbol{u}(t)=\boldsymbol{u}\left(t ; \mathbf{u}_{0}, \varepsilon, \boldsymbol{g}\right)$ of $(2.1)$ with $\boldsymbol{u}\left(t_{0}\right)=\mathbf{u}_{0}$ is defined for all $t \geq t_{0}$ and satisfies the pointwise estimate

$$
|\boldsymbol{u}(t)|_{E} \leq K\left(V_{0}, G_{0}\right)\left(\left|\mathbf{u}_{0}\right|_{E} \mathrm{e}^{-\sigma\left(t-t_{0}\right)}+\int_{t_{0}}^{t} \mathrm{e}^{-\sigma(t-\tau)}|\boldsymbol{g}(\tau)|_{E} d \tau\right)
$$

for every $t \geq t_{0}$. 
In particular, we obtain, for each $\left|\mathbf{u}_{0}\right|_{E} \leq V_{0},|\varepsilon| \leq \varepsilon_{0}$ and $\boldsymbol{g} \in \mathcal{G}_{0}(q)$, the estimate

$$
\left\|\boldsymbol{u}\left(\cdot ; \mathbf{u}_{0}, \varepsilon, \boldsymbol{g}\right)\right\|_{L^{q}\left(t_{0}, T\right)} \leq K\left(\frac{1}{(\sigma q)^{1 / q}}\left|\mathbf{u}_{0}\right|_{E}+\frac{1}{\sigma}\|\boldsymbol{g}\|_{L^{q}\left(t_{0}, T\right)}\right)
$$

for every $T \geq t_{0}$, with $K>0$ given in (2.8), and

$$
\boldsymbol{u}\left(t ; \mathbf{u}_{0}, \varepsilon, \boldsymbol{g}\right) \rightarrow \mathbf{0} \quad \text { as } \quad t \rightarrow \infty
$$

when $1 \leq q<\infty$, or when $q=\infty$ and $\boldsymbol{g}(\infty)=\mathbf{0}$. In a completely similar way, we can switch the role of parameters $\mathbf{u}_{0}$ and $\varepsilon$ in Theorem 2.1 provided that we have $\boldsymbol{f}(t, \mathbf{u})=o\left(|\mathbf{u}|_{E}\right)$ for $\mathbf{u}$ small, uniformly in $t$ :

Theorem 2.2. Let $\mathrm{A}, B(\cdot), \boldsymbol{f}(\cdot, \cdot), \sigma$ be as in (2.2), (2.3) or (2.4), and (2.6), (2.7). Assuming that $\boldsymbol{f}(t, \mathbf{u}) /|\mathbf{u}|_{E} \rightarrow \mathbf{0}$ as $\mathbf{u} \rightarrow \mathbf{0}$, uniformly in $t \geq t_{0}$, then, given any $\varepsilon_{0}, G_{0}>0$, there exist positive constants $K=K\left(\varepsilon_{0}, G_{0}\right), V_{0}=V_{0}\left(\varepsilon_{0}, G_{0}\right)$, depending only on $\mathrm{A}, B, \boldsymbol{f}, \sigma$ and $\varepsilon_{0}, G_{0}$ above, such that, for each $1 \leq q \leq \infty,|\varepsilon| \leq \varepsilon_{0}$ and $\mathbf{u}_{0}, \boldsymbol{g}$ with

$$
\left|\mathbf{u}_{0}\right|_{E} \leq V_{0}\left(\varepsilon_{0}, G_{0}\right), \quad \boldsymbol{g} \in \mathcal{G}_{0}(q):=\left\{\mathbf{g} \in L^{q}\left(t_{0}, \infty\right):\|\mathbf{g}\|_{L^{q}\left(t_{0}, \infty\right)}<G_{0}\right\},
$$

the solution $\boldsymbol{u}(t)=\boldsymbol{u}\left(t ; \mathbf{u}_{0}, \varepsilon, \boldsymbol{g}\right)$ of (2.1) with $\boldsymbol{u}\left(t_{0}\right)=\mathbf{u}_{0}$ is defined for all $t \geq t_{0}$ and satisfies the pointwise estimate

$$
|\boldsymbol{u}(t)|_{E} \leq K\left(\varepsilon_{0}, G_{0}\right)\left(\left|\mathbf{u}_{0}\right|_{E} \mathrm{e}^{-\sigma\left(t-t_{0}\right)}+\int_{t_{0}}^{t} \mathrm{e}^{-\sigma(t-\tau)}|\boldsymbol{g}(\tau)|_{E} d \tau\right)
$$

for every $t \geq t_{0}$.

When $B=0$ in equation (2.1), properties (2.9), (2.10) were obtained in [1], [8] for the particular case $q=2$ via the resolvent method, which uses Laplace transform and Parseval's identity to estimate $L^{2}$ norms. In contrast, our approach is based on the more fundamental estimates (2.8), (2.11), from which (2.9) can be easily derived, allowing arbitrary $q \geq 1$ and broader classes of problems to be considered. The bounds (2.8), (2.11), in turn, follow from even more basic estimates, given in (2.14) below, concerning the fundamental solutions for the linear equation

$$
\boldsymbol{v}_{t}=\mathrm{A} \boldsymbol{v}+B(t) \boldsymbol{v}, \quad t>t_{0},
$$

associated with (2.1), that is, matrix solutions $\Phi(t)$ to the matrix equation

$$
\Phi_{t}=\mathrm{A} \Phi(t)+B(t) \Phi(t), \quad t>t_{0},
$$

with $\Phi\left(t_{0}\right)$ invertible (but otherwise arbitrary).

Theorem 2.3. Assuming (2.2) and (2.3) or (2.4), and choosing $\sigma>0$ as in (2.7), there exists $C>0$ constant (depending only on $\mathrm{A}, B(\cdot), \sigma)$ such that, for each $\tau \geq t_{0}$,

$$
\left\|\Phi(t) \Phi(\tau)^{-1}\right\| \leq C e^{-\sigma(t-\tau)} \quad \forall t \geq \tau,
$$

for any fundamental solution $\Phi(t)$ of (2.12), cf. (2.13). 
We can similarly use (2.14) to get additional results on the solutions of (2.1), as illustrated next. Here, $\varepsilon$ will play no particular role and therefore will be set to 1 . Assume, instead of (2.6), that $\boldsymbol{f}(t, \mathbf{u})$ satisfies, for certain constants $0<\alpha<1$, $\Gamma_{\alpha}>0$, the sublinear growth condition

$$
|\boldsymbol{f}(t, \mathbf{u})|_{E} \leq \Gamma_{\alpha}|\mathbf{u}|_{E}^{\alpha},
$$

for all $\mathbf{u} \in \mathbb{R}^{n}$ and $t \geq t_{0}$, and let $\boldsymbol{u}=\boldsymbol{u}(t)$ be a solution to the equation

$$
\boldsymbol{u}_{t}=\mathrm{A} \boldsymbol{u}+B(t) \boldsymbol{u}+\boldsymbol{f}(t, \boldsymbol{u})+\boldsymbol{g}(t), \quad t>t_{0},
$$

with $\mathrm{A}, B, \boldsymbol{g}, \sigma$ as given in $(2.2)-(2.5),(2.7)$. Then, $\boldsymbol{u}(t)$ is defined for all $t \geq t_{0}$, and uniformly bounded:

Theorem 2.4. Under the assumptions (2.2), (2.3) or (2.4), (2.5), (2.7) and (2.15), there exists a constant $K=K(\alpha, \sigma)>0$, depending only on $\mathrm{A}, B(\cdot), \sigma, \alpha$ and $\Gamma_{\alpha}$, such that the solution $\boldsymbol{u}(t)$ of (2.16) satisfies the estimate

$$
|\boldsymbol{u}(t)|_{E} \leq K(\alpha, \sigma)\left(1+\left|\boldsymbol{u}\left(t_{0}\right)\right|_{E} \mathrm{e}^{-\sigma\left(t-t_{0}\right)}+\int_{t_{0}}^{t} \mathrm{e}^{-\sigma(t-\tau)}|\boldsymbol{g}(\tau)|_{E} d \tau\right),
$$

for all $t \geq t_{0}$.

The distinctive behavior as $\alpha$ crosses its threshold value 1 can be easily checked in the positive solutions of the scalar equation $u_{t}=-u+a u^{\alpha}, a>0$. For $\alpha \neq 1$, these are given by $u(t)=e^{-t}\left(u(0)^{-\beta}-a\left(1-e^{-\beta t}\right)\right)^{-1 / \beta}$, with $\beta:=\alpha-1$. When $\alpha<1$, solutions are bounded for any $a, u(0)>0$ given, but, when $\alpha>1$, solutions blow up at some finite time if $u(0)>(1 / a)^{1 / \beta}$, and decay exponentially fast to $u=0$ $($ as $t \rightarrow \infty)$ if $0<u(0)<(1 / a)^{1 / \beta}$.

\section{Selected Proofs}

In this section we will show the main steps leading to the results described above. Our starting point is (2.14), Theorem 2.3: this is clearly equivalent to the statement that, give any $t_{1} \geq t_{0}$, one has

$$
\|\Phi(t)\| \leq C\left\|\Phi\left(t_{1}\right)\right\| e^{-\sigma\left(t-t_{1}\right)}, \quad \forall t \geq t_{1},
$$

where $C>0$ is the constant in (2.14). To show (3.1), we pick $\tilde{\sigma}>\sigma$ such that $\operatorname{Re} \lambda<-\tilde{\sigma}$ for all $\lambda \in \operatorname{Spec}(\mathrm{A})$, and set $\tilde{C}:=\sup \left\{e^{\tilde{\sigma} t}\left\|e^{t \mathrm{~A}}\right\|: t>0\right\}$. In the case where $B \in L^{p}\left(t_{0}, \infty\right)$ for some $1<p<\infty$, we may proceed as follows: from

$$
\Phi(t)=e^{\left(t-t_{1}\right) \mathrm{A}} \Phi\left(t_{1}\right)+\int_{t_{1}}^{t} e^{(t-\tau) \mathrm{A}} B(\tau) \Phi(\tau) d \tau,
$$

we obtain, using Young's inequality (see e.g. [3], p. 622),

$U(t) \leq \tilde{C}\left\|\Phi\left(t_{1}\right)\right\|+\left(\frac{\tilde{C}}{p}\right)^{p}\left(\frac{p-1}{\tilde{\sigma}-\sigma}\right)^{p-1} \int_{t_{1}}^{t}\|B(\tau)\|^{p} U(\tau) d \tau+(\tilde{\sigma}-\sigma) \int_{t_{1}}^{t} U(\tau) d \tau$, 
where $U(t):=\|\Phi(t)\| e^{\tilde{\sigma}\left(t-t_{1}\right)}$. By Gronwall's lemma, this gives

$U(t) \leq \tilde{C}\left\|\Phi\left(t_{1}\right)\right\| e^{(\tilde{\sigma}-\sigma)\left(t-t_{1}\right)} \tilde{E}_{p}, \quad \tilde{E}_{p}:=\exp \left(\left(\frac{\tilde{C}}{p}\right)^{p}\left(\frac{p-1}{\tilde{\sigma}-\sigma}\right)^{p-1} \int_{t_{0}}^{\infty}\|B(\tau)\|^{p} d \tau\right)$

so that (3.1) holds with $C=\tilde{C} \tilde{E}_{p}$ in the case $p>1$. Similarly, when $p=1$, we set $C_{\mathrm{A}}:=\sup \left\{e^{\sigma t}\left\|e^{t \mathrm{~A}}\right\|: t>0\right\}$, obtaining (3.1) from (3.2) and Gronwall's lemma, with $C$ given this time by

$$
C=C_{\mathrm{A}} \exp \left(C_{\mathrm{A}} \int_{t_{0}}^{\infty}\|B(\tau)\| d \tau\right) .
$$

Finally, in the case where $B \in L^{\infty}\left(t_{0}, \infty\right)$ with $\|B(t)\| \rightarrow 0$ as $t \rightarrow \infty$, we set $\mathcal{B}_{\infty}:=$ $\sup \left\{\|B(t)\|: t>t_{0}\right\}$ and proceed as follows. For $\tilde{\sigma}, \tilde{C}$ defined above, let $T(\tilde{\sigma}) \geq t_{0}$ be sufficiently large so that

$$
\|B(t)\| \leq \frac{\tilde{\sigma}-\sigma}{\tilde{C}}, \quad \forall t \geq T(\tilde{\sigma}) .
$$

If $t_{1} \geq T(\tilde{\sigma})$, we obtain from (3.2) that $V(t):=e^{\tilde{\sigma}\left(t-t_{1}\right)}\left\|\Phi(t) \Phi\left(t_{1}\right)^{-1}\right\|$ satisfies

$$
V(t) \leq \tilde{C}+(\tilde{\sigma}-\sigma) \int_{t_{1}}^{t} V(\tau) d \tau, \quad \forall t \geq t_{1},
$$

so that (3.1) holds with $C=\tilde{C}$. If $t_{0} \leq t_{1}<T(\tilde{\sigma})$, we have, for $V(t)$ given above,

$$
V(t) \leq \tilde{C}+\tilde{C} \int_{t_{1}}^{T(\tilde{\sigma})} \mathcal{B}_{\infty} V(\tau) d \tau+(\tilde{\sigma}-\sigma) \int_{T(\tilde{\sigma})}^{t} V(\tau) d \tau
$$

for any $t \geq T(\tilde{\sigma})$, so that, by Gronwall's lemma, we obtain

$$
V(t) \leq \tilde{C} \exp \left(\tilde{C} \mathcal{B}_{\infty}\left(T(\tilde{\sigma})-t_{0}\right)\right) \exp \left((\tilde{\sigma}-\sigma)\left(t-t_{1}\right)\right),
$$

which shows (3.1) with $C:=\tilde{C} \exp \left(\tilde{C} \mathcal{B}_{\infty}\left(T(\tilde{\sigma})-t_{0}\right)\right)$. Finally, for $t_{1} \leq t \leq T(\tilde{\sigma})$, we obtain, from (3.2) and Gronwall's lemma,

$$
e^{\sigma\left(t-t_{1}\right)}\|\Phi(t)\| \leq C_{\mathrm{A}} \| \Phi\left(t_{1} \| \exp \left(C_{\mathrm{A}} \mathcal{B}_{\infty}\left(T(\tilde{\sigma})-t_{0}\right)\right),\right.
$$

giving (3.1) with $C:=C_{\mathrm{A}} \exp \left(C_{\mathrm{A}} \mathcal{B}_{\infty}\left(T(\tilde{\sigma})-t_{0}\right)\right)$, and the proof is complete.

Having established Theorem 2.3, we can now derive the fundamental estimates given in Theorems 2.1, 2.2 and 2.4 above. Starting with (2.8), let $\Phi(t)$ be a fundamental matrix solution to (2.12), take $\sigma>0$ as in (2.7) and choose $\tilde{\sigma}>\sigma$ so that $\operatorname{Re} \lambda<-\tilde{\sigma}$ for every $\lambda \in \operatorname{Spec}(\mathrm{A})$. Using (2.15), set $\tilde{C} \in[1, \infty[$ by

$$
\tilde{C}:=\sup _{t \geq \tau \geq t_{0}} e^{\tilde{\sigma}(t-\tau)}\left\|\Phi(t) \Phi(\tau)^{-1}\right\|
$$

and let $\Gamma_{0}>0$ be large enough so that

$$
|\boldsymbol{f}(t, \mathbf{u})|_{E} \leq \Gamma_{0}|\mathbf{u}|_{E} \quad \text { if }|\mathbf{u}|_{E} \leq \gamma_{0}:=2 \tilde{C}\left(V_{0}+(1+1 / \sigma) G_{0}\right) .
$$


Setting

$$
\varepsilon_{0}:=\frac{\tilde{\sigma}-\sigma}{2 \Gamma_{0} \tilde{C}}
$$

and assuming $\left|\mathbf{u}_{0}\right|_{E} \leq V_{0},|\varepsilon| \leq \varepsilon_{0}$ and $\boldsymbol{g} \in \mathcal{G}_{0}(q)$, i.e., $\boldsymbol{g} \in L^{q}\left(t_{0}, \infty\right), 1 \leq q \leq \infty$, with $\|\boldsymbol{g}\|_{L^{q}\left(t_{0}, \infty\right)} \leq G_{0}$, the solution $\boldsymbol{u}(t)=\boldsymbol{u}\left(t ; \mathbf{u}_{0}, \varepsilon, \boldsymbol{g}\right)$ of $(2.1)$ with $\boldsymbol{u}\left(t_{0}\right)=\mathbf{u}_{0}$ satisfies, for $t>t_{0}$,

$\boldsymbol{u}(t)=\Phi(t) \Phi\left(t_{0}\right)^{-1} \mathbf{u}_{0}+\int_{t_{0}}^{t} \Phi(t) \Phi(\tau)^{-1} \boldsymbol{g}(\tau) d \tau+\varepsilon \int_{t_{0}}^{t} \Phi(t) \Phi(\tau)^{-1} \boldsymbol{f}(\tau, \boldsymbol{u}(\tau)) d \tau$,

while $|\boldsymbol{u}(\tau)|_{E}<\gamma_{0}, t_{0}<\tau<t$, is verified. This gives, by (2.14) and (3.3)-(3.5),

$|\boldsymbol{u}(t)|_{E} \leq \tilde{C} e^{-\tilde{\sigma}\left(t-t_{0}\right)}\left|\mathbf{u}_{0}\right|_{E}+\tilde{C} \int_{t_{0}}^{t} e^{-\tilde{\sigma}(t-\tau)}|\boldsymbol{g}(\tau)|_{E} d \tau+\varepsilon \Gamma_{0} \tilde{C} \int_{t_{0}}^{t} e^{-\tilde{\sigma}(t-\tau)}|\boldsymbol{u}(\tau)|_{E} d \tau$,

so that, setting $U(t):=e^{\sigma\left(t-t_{0}\right)}|\boldsymbol{u}(t)|_{E}$, we obtain

$$
U(t) \leq \tilde{C}\left|\mathbf{u}_{0}\right|_{E}+\tilde{C} \int_{t_{0}}^{t} e^{\sigma\left(\tau-t_{0}\right)}|\boldsymbol{g}(\tau)|_{E} d \tau+\varepsilon \Gamma_{0} \tilde{C} \int_{t_{0}}^{t} e^{-(\tilde{\sigma}-\sigma)(t-\tau)} U(\tau) d \tau
$$

By a Gronwall-type argument, it follows that

$$
U(t) \leq \tilde{C}\left(\left|\mathbf{u}_{0}\right|_{E}+\int_{t_{0}}^{t} e^{\sigma\left(\tau-t_{0}\right)}|\boldsymbol{g}(\tau)|_{E} d \tau\right) \frac{\tilde{\sigma}-\sigma}{\tilde{\sigma}-\sigma-\varepsilon \Gamma_{0} \tilde{C}}
$$

or, because $\varepsilon_{0} \Gamma_{0} \tilde{C} \leq(\tilde{\sigma}-\sigma) / 2$,

$$
|\boldsymbol{u}(t)|_{E} \leq 2 \tilde{C}\left(\left|\mathbf{u}_{0}\right|_{E} e^{-\sigma\left(t-t_{0}\right)}+\int_{t_{0}}^{t} e^{\sigma\left(\tau-t_{0}\right)}|\boldsymbol{g}(\tau)|_{E} d \tau\right)
$$

in particular,

$$
|\boldsymbol{u}(t)|_{E} \leq 2 \tilde{C}\left(V_{0} e^{-\sigma\left(t-t_{0}\right)}+\left(\frac{1}{\sigma}\right)^{1-1 / q} G_{0}\right)<\gamma_{0},
$$

so that $\boldsymbol{u}(t)$ is defined for all $t \geq t_{0}$ and (2.8) must be valid for $\boldsymbol{u}(t)$ with $K:=2 \tilde{C}$. A similar argument is used to show (2.11), (2.17).

\section{Concluding Remarks}

In this work, we derived a number of fundamental solution estimates to a broad class of nonlinear systems of the form (2.1) that satisfy our main assumptions (2.2) and (2.3) or (2.4). The estimates given in (2.8), (2.9), (2.10), (2.11), (2.14) and (2.17) provide very useful extensions of classical results such as the Poincaré-Lyapunov stability theorem for weakly nonlinear systems or the $L^{2}$ results discussed in [1], [8] for ODE systems like (2.1). Indeed, Theorems $2.1-2.4$ give fairly complete statements that should prove convenient to use in most applications concerning properties related to those treated here. For example, let $\boldsymbol{u}(\cdot ; \mathbf{0}, \boldsymbol{g}), \boldsymbol{u}(\cdot ; \mathbf{0}, \hat{\boldsymbol{g}})$ be solutions of (2.16), with zero initial values, corresponding to given functions $\boldsymbol{g}, \hat{\boldsymbol{g}} \in \mathcal{G}_{0}(q)$ for some $G_{0}>0,1 \leq q \leq \infty$. Assuming $\boldsymbol{f} \in C^{1}$ with bounded $\mathbf{u}$-derivatives for 
bounded $\mathbf{u} \in \mathbb{R}^{n}$ and all $t \geq t_{0}$, and that $\boldsymbol{f}(t, \mathbf{u})=o\left(|\mathbf{u}|_{E}\right)$ for $|\mathbf{u}|_{E}$ small, uniformly in $t$, it follows from Theorems 2.2 and 2.3 that $\boldsymbol{u}(\cdot ; \mathbf{0}, \boldsymbol{g}), \boldsymbol{u}(\cdot ; \mathbf{0}, \hat{\boldsymbol{g}}) \in L^{q}\left(t_{0}, \infty\right)$ and, for some constant $C=C\left(G_{0}\right)$ that depends only on $G_{0}$ (and the functions A, $B, \boldsymbol{f}$ ) but not on $\boldsymbol{g}, \hat{\boldsymbol{g}} \in \mathcal{G}_{0}(q)$, we have

$$
\|\boldsymbol{u}(\cdot ; \mathbf{0}, \boldsymbol{g})-\boldsymbol{u}(\cdot ; \mathbf{0}, \hat{\boldsymbol{g}})\|_{L^{q}\left(t_{0}, \infty\right)} \leq C\left(G_{0}\right)\|\boldsymbol{g}-\hat{\boldsymbol{g}}\|_{L^{q}\left(t_{0}, \infty\right)} .
$$

Likewise, other properties of interest can be similarly addressed.

\section{Referências}

[1] P.A. Braz e Silva, "Stability of plane Couette flow: the resolvent method", PhD Thesis, University of New Mexico, Albuquerque, NM, USA, 2002.

[2] E.A. Coddington and N. Levinson, "Theory of Ordinary Differential Equations", McGraw-Hill, New York, 1955.

[3] L.C. Evans, "Partial Differential Equations", American Mathematics Society, Providence, 1998.

[4] T.H. Gronwall, Note on the derivatives with respect to a parameter of the solutions of a system of differential equations, Ann. Math., 20 (1919), 292-296.

[5] T. Hagstrom, H.-O. Kreiss, J. Lorenz and P. Zingano, Decay in time of incompressible flows, J. Math. Fluid Mech., 5 (2003), 231-244.

[6] E. Hairer, S.P. Norsett and G. Wanner, "Solving Ordinary Differential Equations", Vol. I, Springer, Berlin, 1987.

[7] J. Hale, "Ordinary Differential Equations", Wiley, New York, 1969.

[8] H.O. Kreiss and J. Lorenz, Resolvent estimates and quantification of nonlinear stability, Acta Math. Sin., 16 (2000), 1-20.

[9] A.M. Lyapunov, "The General Problem of Stability of Motion" (Russian), 1892, reprinted by Princeton Univ. Press, Princeton, 1947.

[10] H. Poincaré, "Les Méthodes Nouvelles de la Mécanique Céleste", GauthierVillars, Paris, 1893.

[11] E.J. Routh, "A Treatise on the Stability of a given State of Motions", Adams Prize essay, Cambridge University, Cambridge, 1877.

[12] F. Verhulst, "Nonlinear Differential Equations and Dynamical Systems," Springer, Berlin, 1990. 
